\title{
The influence and countermeasures of the application of blockchain on the investment efficiency of cultural industry DEA Analysis of listed companies based on blockchain concept
}

\author{
Jian-bo $\mathrm{LI}^{1, \mathrm{a}}$, Rui ZHANG ${ }^{2, \mathrm{~b}}$,Yue $\mathrm{YIN}^{3, \mathrm{c}, *}$ \\ ${ }^{1}$ Dalian Polytechnic University, Dalian, China \\ ${ }^{2}$ Dalian Polytechnic University, Dalian, China \\ ${ }^{3}$ Dalian Polytechnic University, Dalian, China
}

\begin{abstract}
Block chain technology has changed the traditional digital economy, promoted the formation of a new value and trust system, and at the same time triggered the transformation of cultural industry, providing a means to improve investment efficiency. This paper takes the listed companies of cultural industry in the concept of block chain as the research sample, and uses DEA efficiency evaluation method to empirically analyze the change of cultural industry investment efficiency. The results show that: block chain technology has an impact on pure technical efficiency and also promotes profit growth. However, the development and application of block chain technology need to be more integrated with cultural industry investment to open up a new development path for the traditional information society.
\end{abstract}

\section{Foreword}

With the development and promotion of digital economy, block chain technology, as an emerging technology, has brought subversive innovation changes to the economic society with the characteristics of decentralization, reliability, anonymity and unforgeability. Block chain technology also brings challenges to the business model, technology research and development, and government regulation of relevant industries (Li Xiaomei, 2019). In order to encourage the $R \& D$ and application of block chain technology and realize the integration of digital economy and real economy, China has successively introduced corresponding policies and regulations. In December 2016, the 13th five year National informatization Plan of the State Council clearly pointed out for the first time that the development of cutting-edge technologies such as block chain should be strengthened; in January 2019, the state Internet Information Office (hereinafter referred to as the office of state information technology) issued the regulations on the management of block chain information services, aiming to standardize and promote the healthy development of block chain technology and related services. However, how to correctly apply block chain to cultural industry investment, improve investment efficiency, break through the bottleneck of integration, and promote the healthy development of China's cultural industry, is worthy of in-depth discussion.

\section{A review of the literature}

Since the concept of block chain was first proposed in 2008, its development has experienced a great change from prominence to popularity (Zhang Yueling, 2019).Based on the anonymity characteristics, block chain technology provides a new way for privacy data protection (Swan, 2015). In medical issues, medshare system uses the data traceability ability of block chain technology to solve the sharing problem of cloud storage medical data (Xia qi, 2017). Comparatively speaking, domestic research started late, but the development trend is good. Chinese scholar Hou Yanhong (2008) analyzed the reasons for the poor input-output effect of Tianjin's cultural industry by constructing DEA model and EVA model from the meso and micro perspectives, and put forward relevant countermeasures. Zhang Guiling (2016) used DEA to analyze the investment and financing efficiency of cultural industry in 31 provinces and cities in mainland China. However, the domestic empirical research mainly stays at the level of government policy research, and rarely goes deep into the field of technological innovation and cultural industry enterprises. Therefore, the combination of cultural industry and block chain technology and the impact of block chain on the investment efficiency of cultural industry will become a hot topic in the future academic research. 


\section{DEA Analysis on investment efficiency of Listed Companies in blockchain concept cultural industry -- a case study of 12 listed companies}

\subsection{Technical efficiency measurement of 12 listed companies in cultural industry}

\subsubsection{Efficiency measurement index}

Although the cultural industry is a sunrise industry in the 21 st century, the low efficiency of investment and financing has always been a common phenomenon in this industry. DEA (Data Envelopment Analysis) is a method that is suitable for the evaluation of relative efficiency and benefit of the same type of multiple input multiple output decision-making units, which was put forward by Charnes and Coop-er in 1978. In order to better evaluate the investment efficiency of Listed Companies in the block chain concept cultural industry, DEA indicators are set from two aspects: acquisition ability and utilization capacity, namely input and output. Input refers to the economic volume in economic activities of Listed Companies in each year, and output refers to the economic quantity of output effect of listed companies under input factors. This paper first selects 199 "block chain" concept stocks from Shanghai and Shenzhen A-shares, and then finds 12 stocks such as Storm Group and blue cursor through the industry ownership of "cultural media", and uses DEA method to study and analyze 2016-2018 The financial report of cultural industry company introduced block chain project in, analyzes the comprehensive efficiency, pure technical efficiency and scale efficiency, and observes the change of investment efficiency, so as to verify the feasibility of the integration of block chain technology and cultural industry investment.

The selected indicators are shown in Table 1.

Table 1. Input and output index selection

\begin{tabular}{ccc}
\hline Input / output index & financial index & Origin or standard \\
\hline Input index & total assets & "total assets" in the balance sheet or through \\
& "liabilities + owner's equity" & total liabilities / total assets \\
& Asset liability ratio & Quick ratio \\
& Return on equity & net profit / net assets \\
Output index & Operating revenue growth rate & operating revenue growth amount / operating \\
& Total assets turnover & revenue in the base period \\
& net operating income / average total assets \\
\hline
\end{tabular}

\subsubsection{Technical efficiency measurement}

Input index (total assets, quick ratio and asset liability ratio) and output index (return on net assets, growth rate of operating income and turnover rate of total assets) are input into deap2.1 software for measurement, and BCC scale income variable model is used for analysis to obtain the technical efficiency of each enterprise (see Table 2)

Table 2. Technical efficiency of 12 listed companies in cultural industry in different year

\begin{tabular}{|c|c|c|c|c|}
\hline enterprise & particular year & comprehensive efficiency & pure technical efficiency & scale efficiency \\
\hline Storm & 2016 & $\overline{1}$ & 1 & 1 \\
\hline \multirow{2}{*}{ Group } & 2017 & 1 & 1 & 1 \\
\hline & 2018 & 1 & 1 & 1 \\
\hline Blue & 2016 & 0.823 & 1 & 0.823 \\
\hline \multirow{2}{*}{ Cursor } & 2017 & 0.846 & 0.856 & 0.988 \\
\hline & 2018 & 1 & 1 & 1 \\
\hline Xinhua & 2016 & 1 & 1 & 1 \\
\hline \multirow[t]{2}{*}{ Wenxuan } & 2017 & 0.932 & 0.989 & 0.942 \\
\hline & 2018 & 0.9 & 0.964 & 0.934 \\
\hline Zhejiang & 2016 & 1 & 1 & 1 \\
\hline Digital & 2017 & 0.715 & 0.864 & 0.827 \\
\hline Culture & 2018 & 0.763 & 0.764 & 0.999 \\
\hline Jinke & 2016 & 1 & 1 & 1 \\
\hline \multirow[t]{2}{*}{ Culture } & 2017 & 1 & 1 & 1 \\
\hline & 2018 & 0.849 & 0.881 & 0.963 \\
\hline Vision & 2016 & 0.947 & 0.965 & 0.981 \\
\hline \multirow[t]{2}{*}{ China } & 2017 & 1 & 1 & 1 \\
\hline & 2018 & 1 & 1 & 1 \\
\hline Keda & 2016 & 1 & 1 & 1 \\
\hline \multirow[t]{2}{*}{ Share } & 2017 & 0.987 & 1 & 0.987 \\
\hline & 2018 & 1 & 1 & 1 \\
\hline Huamei & 2016 & 1 & 1 & 1 \\
\hline \multirow[t]{2}{*}{ Holding } & 2017 & 0.964 & 0.984 & 0.979 \\
\hline & 2018 & 0.952 & 0.979 & 0.973 \\
\hline Xinhuanet & 2016 & 0.928 & 0.975 & 0.951 \\
\hline
\end{tabular}




\begin{tabular}{ccccc} 
& 2017 & 0.781 & 0.799 & 0.978 \\
Tianzhou & 2018 & 0.744 & 0.753 & 0.988 \\
Culture & 2016 & 1 & 1 & 1 \\
& 2017 & 1 & 1 & 1 \\
Chinese & 2018 & 1 & 1 & 1 \\
On-line & 2016 & 1 & 1 & 1 \\
& 2017 & 1 & 1 & 1 \\
Kaiying & 2018 & 1 & 1 & 1 \\
Network & 2016 & 1 & 1 & 1 \\
& 2017 & 1 & 0.956 & 0.964 \\
\hline
\end{tabular}

It can be seen from table 2 that the comprehensive efficiency, pure technical efficiency and scale efficiency of Vision China show an upward trend from 2016 to 2018. Through the study of the annual report of Vision China, we can find that: from 2016 to 2018 , the R\&D investment of Vision China was showing an upward trend year by year. From 2016 to 2018, from Blue Cursor, the comprehensive efficiency and scale efficiency showed an upward trend, and the pure technical efficiency showed a "V" type change. In the remaining 10 listed companies, excluding the three listed companies which have not changed in comprehensive efficiency, pure technical efficiency and scale efficiency from 2016 to 2018, and Keda Share with V-shaped change, it is found that the scale efficiency of Zhejiang Digital Culture, Jinke Culture, Xinhuanet and Kaiying Network in 2018 is greater than Technical efficiency $>$ total efficiency. The scale efficiency of Xinhua Wenxuan and Huame Holding is slightly lower than the technical efficiency, which indicates that the technology development of block chain of these companies has not had a significant effect on the comprehensive efficiency in a short time, and the observation period needs to be lengthened.

\subsection{Research conclusion}

\subsubsection{Blockchain technology has an impact on pure technical efficiency}

From 2016 to 2018, there were 4 companies with no change in pure technical efficiency (Storm Group, Keda Holding, Tianzhou Culture and Chinese On-line); the pure technical efficiency of Xinhuanet decreased year by year from 2016 to 2018; there were 3 companies (Xinhua Wenxuan, Zhejiang Digital Culture and Huamei Holding) that had changed in the two years from 2017 to 2018; and 4 companies whose pure technical efficiency deviated from 1 in only one year Vision China (2016), Blue Cursor (2017), Jinke Culture (2018) and Kaiying Network (2018), the deviation of technical efficiency reflects the change of enterprise R\&D process.

\subsubsection{Blockchain technology promotes enterprise profit growth}

In Table 2, Blue Cursor listed companies with good integration effect of block chain established strategic cooperation relationship with DATA Foundation in 2018, and established marketing block chain aboratory of Blue Cursor Kunluntang Research Institute. In May 2018, Blue
Cursor obtained 87 software copyrights and 19 technology patents, and the revenue generated automatically by the machine has reached the scale of about 2 million yuan per day,which also avoided cheating and fraud in traditional advertising.

\section{Conclusion}

At present, Blue Cursor and other cultural industry listed companies have gradually integrated with the block chain field. Based on the existing problems of cultural industry investment, this paper puts forward the following suggestions on the integration of block chain into the investment of listed companies in cultural industry:on the capital level, the biggest obstacle of cultural industry investment from the perspective of block chain is the stability of capital integration. Relying on the block chain technology, the investment and financing parties of cultural industry can be connected point-to-point without communication through intermediary, which can avoid complicated financing procedures and high financing costs.Technical level:ensure the security of cultural industry investment objects, make full use of the unforgeable and irreversible characteristics of block chain technology data generation, encrypt and share the contract,content and other information of cultural industry investment, so as to form a set of true and reliable information base,which can reflect the time sequence of information records and can not be tampered with.After the completion of the investment project,according to the real data provided by the block chain,evaluate the investment efficiency and potential risks of the investment project,and form a summary report or rules and regulations to provide reference for improving the investment efficiency of cultural industry.

\section{References}

1. Li Xiaomei, Xia Maosen. Measurement and improvement path of input-output efficiency of strategic emerging industry enterprises in Liaoning Province: Based on the sample data of Listed Companies in Liaoning Province 2012-2016[J]. Science and technology management research,2019, 39(2):80-85.

2. Zhang Yueling,Wang Xiaojing.An analysis of online audit framework in the context of blockchain technology[J].Audit abstracts,2019(4):111-116. 
3. SWAN M.Block chain thinking: the brain as a decen-tralized autonomous corporation[commentary] [J].IEEE Technology and Society Magazine, 2015, 34(4):41-52.

4. XIA Q I,SIFAN E B,ASAMOAH K O,et al. MeD-Share:trust-less medical data sharing among cloud service providers via block chain[J].IEEE Access, 2017,5:14757-14767.

5. Hou Yanhong.Research on performance evaluation of cultural industry input [D].Tianjin::Tianjin University of technology,2008.

6. Zhang Guiling. Research on investment and financing efficiency of cultural industry based on DEA [J].Friends of accounting,2016,(21). 